\title{
Genome Editing Technologies as Cellular Defense Against Viral Pathogens
}

\author{
Yingzi Zhang' and Mo Li*t \\ Biological and Environmental Sciences and Engineering Division (BESE), King Abdullah University of Science and Technology \\ (KAUST), Thuwal, Saudi Arabia
}

OPEN ACCESS

Edited by:

Junjiu Huang,

Sun Yat-sen University, China

Reviewed by:

Yong Fan,

Guangzhou Medical University, China

Feng Gu,

Wenzhou Medical University, China

${ }^{*}$ Correspondence:

Mo Li

mo.li@kaust.edu.sa

tORCID:

Yingzi Zhang

orcid.org/0000-0001-6871-5406

Mo $\mathrm{Li}$

orcid.org/0000-0003-0827-8907

Specialty section:

This article was submitted to

Cellular Biochemistry,

a section of the journal

Frontiers in Cell and Developmental

Biology

Received: 28 May 2021

Accepted: 21 June 2021

Published: 15 July 2021

Citation:

Zhang Y and Li M (2021) Genome Editing Technologies as Cellular

Defense Against Viral Pathogens. Front. Cell Dev. Biol. 9:716344. doi: 10.3389/fcell.2021.716344
Viral infectious diseases are significant threats to the welfare of world populations. Besides the widespread acute viral infections (e.g., dengue fever) and chronic infections [e.g., those by the human immunodeficiency virus (HIV) and hepatitis B virus (HBV)], emerging viruses, such as severe acute respiratory syndrome coronavirus 2 (SARSCoV-2), pose great challenges to the world. Genome editing technologies, including clustered regularly interspaced short palindromic repeats (CRISPR)-CRISPR-associated (Cas) proteins, zinc-finger nucleases (ZFNs), and transcription activator-like effector nucleases (TALENS), have played essential roles in the study of new treatment for viral infectious diseases in cell lines, animal models, and clinical trials. Genome editing tools have been used to eliminate latent infections and provide resistance to new infections. Increasing evidence has shown that genome editing-based antiviral strategy is simple to design and can be quickly adapted to combat infections by a wide spectrum of viral pathogens, including the emerging coronaviruses. Here we review the development and applications of genome editing technologies for preventing or eliminating infections caused by HIV, HBV, HPV, HSV, and SARS-CoV-2, and discuss how the latest advances could enlighten further development of genome editing into a novel therapy for viral infectious diseases.

Keywords: genome editing, viral infectious disease, CRISPR/Cas, SARS-CoV-2, HIV, hepatitis B, emerging pathogen

\section{INTRODUCTION}

Viral infectious diseases are significant threats to human well-being and a global economic burden (King et al., 2006). Though the life cycle of different viruses may vary, it typically starts with the attachment of viruses to target cells and is followed by cellular entry, uncoating to release viral contents, replication and biosynthesis guided by the viral genome, assembly of new viral particles, and release of virions. By completing its life cycle, the virus multiplies and can infect more cells. Besides, many viral pathogens can establish latent or chronic infections by integrating their genome into the genome of the host cell (Figure 1). Since the integrated viral genome, called provirus, remains dormant in the host cell and its daughter cells, it can evade humoral immunity or antiviral treatment and become reactivated and assemble new virions under favorable conditions. Thus, despite of the development of antiviral therapy, chronic infection is still uncurable for many viral 


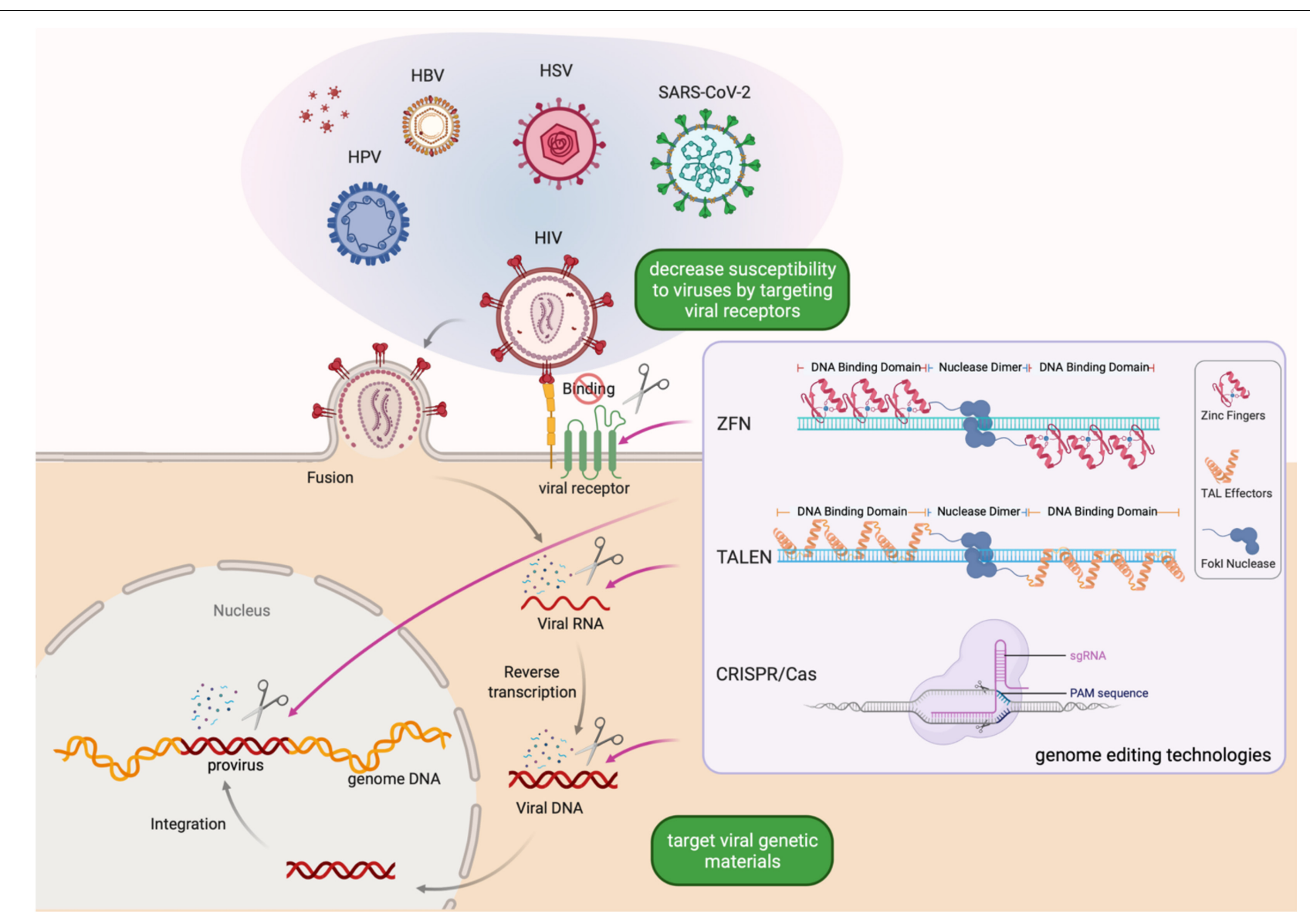

FIGURE 1 | Schematic of genome editing strategies for preventing or eliminating viral infections. Genome editing-based antiviral strategy is simple to design and can be quickly adapted to combat infection by a wide-spectrum of viral pathogens, aiming to eliminate latent infections and provide resistance to new infections. Created with BioRender.com.

pathogens (Trickey et al., 2017; Kulkarni et al., 2020; Pujanandez, 2020). Effective treatment options are currently absent for many viral infectious diseases.

The emergence of genome editing technologies enables researchers to precisely manipulate specific genomic sequences. By adding, removing, or altering specific DNA sequences in the genome, genome editing technologies offer new solutions for the treatment of viral infectious diseases in the clinic. The mainstream genome editing technologies include zinc finger nucleases (ZFNs), transcriptional activator-like effector nucleases (TALENs), and clustered regularly interspersed short palindromic repeats and CRISPR-associated proteins (CRISPR/Cas) (Li et al., 2014). Genome editing-based strategies for combating viral infectious diseases vary according to the characteristics of the virus and the host (Figure 1). For example, some human proteins are co-opted as viral receptors, and thus can be targets of genome editing ( $\mathrm{Li}$ et al., 2014; Tebas et al., 2014; Xu et al., 2019). ZFNs were the first designer nucleases used to modify the CCR5 gene, a chemokine receptor on immune cells co-opted by the HIV as a co-receptor of cellular entry, to prevent HIV infection. The CRISPR/Cas system, thanks to its ease of use and versatility, has become the dominant technology in genome editing (Anzalone et al., 2020). Various in vitro and animal model experiments have been performed to reduce or eliminate viral infections using the CRISPR/Cas system. Clinical studies of antiviral therapy based on CRISPR/Cas showed great promises (Tebas et al., 2014; Gupta et al., 2019; Xu et al., 2019; Anzalone et al., 2020). Here, we first offer an overview of genome editing technologies and then provide a timely update on the development of genome editing therapy for common viral infectious diseases.

\section{MAINSTREAM GENOME EDITING TECHNOLOGIES}

All three mainstream genome editing technologies are based on designer nucleases that exploit DNA sequence specific recognition mechanisms existing in nature. ZFNs were invented in the 1990's and deployed as the first bona fide designer nuclease for genome editing (Kim et al., 1996; Bitinaite et al., 1998; Porteus and Carroll, 2005; Urnov et al., 2010). A ZFN consists of an array of zinc finger DNA binding motifs and a FokI endonuclease domain. Each zinc finger motif recognizes 
three nucleotides. Two ZFNs, each containing a tandem array of 3-6 zinc finger motifs that recognize half of the target site, are required for DNA cleavage. Upon recognition of the binding sites, the FokI domains of the pair of ZFNs are placed in close proximity and become activated to cleave the DNA in the middle of the two half sites (Figure 1). As the pioneering genome editing technology, ZFNs facilitated targeted introduction of desired changes in genetic materials in different organisms. However, one main concern associated with the use of ZFNs is that they may lead to off-target mutations (Porteus and Baltimore, 2003; Alwin et al., 2005; Szczepek et al., 2007). To address this problem, researchers developed several approaches, including obligate heterodimeric ZFN architectures and protein-engineering methods, to enhance the specificity of ZFNs. However, it is time-consuming to design ZFNs and empirical testing is necessary to screen for ZFNs with sufficient activity and specificity (Li et al., 2014). These constraints limit the use of ZFNs in high-throughput genome editing.

The rising interest in genome editing beckoned a rapid development of new technologies. Like the design principles of ZFNs, TALENs consist of a transcriptional activator-like effector (TALE) repeat domain and a FokI nuclease domain (Figure 1). Each TALE effector repeat has two amino acids termed repeatvariable di-residues that determine its specificity for one base pair (Boch et al., 2009). As TALEs are evolved to function in tandem arrays, the modular assembly of TALENs has a much higher success rate than that of ZFNs. As such, the TALEN technology is simpler and more economical to deploy, while maintaining a high specificity, making it feasible to realize high throughput genome editing (Li et al., 2014).

The CRISPR/Cas system, whose discovery involved two decades of research by many researchers (Lander, 2016), has quickly become the most popular genome editing technology since 2012. In 1987, Ishino et al. (1987) identified the CRISPR locus when they discovered a genetic structure containing five highly homologous 29-nucleotide repeats separated by 32-nucleotide spacers. Derived from invading mobile genetics elements (MGEs), the spacer sequences are leveraged by bacteria and archaea to form an adaptive immune system (Strich and Chertow, 2019). The bacterial CRISPR system can be programmed to function in other species where a CRISPR RNA (crRNA) complementary to the target DNA and a trans-activating RNA (tracrRNA) guide the Cas nuclease to induce DNA double-strand breaks (Figure 1), followed by repair by either non-homologous end joining or homologydirected repair (Garneau et al., 2010; Deltcheva et al., 2011; Jiang and Doudna, 2017).

A diverse range of CRISPR/Cas systems with different characteristics have been described to date. For example, the CRISPR/Cas9 system targets DNA, while the CRISPR/Cas13 system targets RNA. Besides, while CRISPR/Cas9 requires the NGG protospacer adjacent motif (PAM), CRISPR/Cas13 (e.g., from Leptotrichia wadei) has no requirement for protospacer flanking sequences (Abudayyeh et al., 2017), which offers flexibility when targeting viruses with rapidly emerging mutations and new variants. For a detailed discussion on CRISPR/Cas genome editing tools and considerations for choosing the right tool for the application, the readers are referred to an excellent recent review (Anzalone et al., 2020).

Genome editing technologies depend on effective delivery strategies to have a desirable effect on the target biological system. Lentivirus and adeno-associated virus (AAV) are two widely utilized viral vectors for delivering genetic materials. Lentiviral vectors are easy to package and have a larger payload size than AAV vectors. An important caveat of lentiviral vectors is that they integrate into the genome of the target cell. Such permanent alterations of the host genome could potentially limit their application in basic research and in the clinic. In this regard, AAV vectors, which normally non-integrative, are generally considered to have a more desirable safety profile under normal circumstances (Athanasopoulos et al., 2017). Although recently, AAV vectors have been reported to integrate into DSBs induced by CRISPR/Cas9, raising the awareness of the risk of insertional mutagenesis when AAV is used with CRIPSR/Cas (Nault et al., 2015; Hanlon et al., 2019). Moreover, AAVs have various serotypes specific to different organs, which can be useful to target specific tissues. AAV vectors are widely used both in basic research and in clinical trials. While studies are looking into the effectiveness and safety of viral vectors, non-viral vectors are also being explored as alternatives (see Conclusion and Perspectives).

\section{GENOME EDITING TECHNOLOGIES AS NOVEL ANTIVIRAL DEFENSE}

The utilization of ZFNs, TALENs, and CRISPR/Cas9 on viral infectious diseases requires the target virus to exist in a DNA form during at least part of its life cycle (Figure 1). Luckily, the major viruses that threaten human health worldwide, including human immunodeficiency virus type 1 (HIV-1), human papillomavirus (HPV), and herpes simplex virus (HSV) type 1 and 2, satisfy this requirement.

\section{Development of Genome Editing Therapy for HIV}

HIV is a global public health burden, infecting an estimated 38 million people at the end of 2019. In HIV-1-infected patients who do not have detectable viral replication, there may still be about $10^{7} \mathrm{CD}^{+} \mathrm{T}$ cells latently infected (Blankson et al., 2014). Because of this, although current small-molecule antiretroviral therapy (ART) may effectively inhibit HIV replication, it cannot fully eliminate the virus (Trickey et al., 2017; Kulkarni et al., 2020). Studies have shown that genome editing-based therapies can target both the active and latent HIV-1 infections (Figure 1; Hu W. et al., 2014; Yin C. et al., 2017; Yin et al., 2020).

There are different strategies to target HIV using genome editing technologies. One of them is to establish CCR5-null cells that deny HIV-1 entry (Table 1). In a pioneering study published in 2014, Tebas et al. (2014) utilized ZFN-mediated knockout of the CCR5 gene in autologous CD4 T cells of six persons infected with HIV. The clinical trial proved the safety of ZFNbased therapy within the limits of the study, and showed the feasibility and efficiency to decrease the blood level of HIV-1 
TABLE 1 | Recent studies of genome editing therapy for viral infectious diseases.

\begin{tabular}{|c|c|c|c|c|}
\hline Virus & Context & $\begin{array}{l}\text { Genome editing } \\
\text { technology }\end{array}$ & Strategy & References \\
\hline \multirow[t]{8}{*}{ HIV } & Autologous CD4 T cells in people & ZFN & $\begin{array}{l}\text { Lead to a five-nucleotide duplication } \\
\text { modification (pentamer) in CCR5 }\end{array}$ & $\begin{array}{l}\text { Tebas et al., } \\
2014\end{array}$ \\
\hline & $\begin{array}{l}\text { HeLa-derived TZM-bl cells. Latently infected } \\
\text { microglial, promonocytic, and T cells }\end{array}$ & CRISPR/Cas9 & $\begin{array}{l}\text { Excise a 9,709-bp fragment of integrated } \\
\text { proviral DNA spanning from its } 5^{\prime} \text { to } 3^{\prime} \text { LTRs }\end{array}$ & $\begin{array}{l}\text { Hu W. et al., } \\
2014\end{array}$ \\
\hline & Three different animal models & CRISPR/Cas9 & $\begin{array}{l}\text { A quadruplex cocktail strategy to lead to } \\
\text { multiplex fragmental deletions and multiple indel } \\
\text { mutations in the HIV-1 provirus }\end{array}$ & $\begin{array}{l}\text { Yin C. et al., } \\
2017\end{array}$ \\
\hline & $\begin{array}{l}\text { Infected human peripheral blood mononuclear cells } \\
\text { within transgenic mouse models }\end{array}$ & CRISPR/Cas9 & $\begin{array}{l}\text { Remove the proviral DNA fragment from the } \\
\text { HIV-1 viral genome within the LTRs }\end{array}$ & $\begin{array}{l}\text { Bella et al., } \\
2018\end{array}$ \\
\hline & $\begin{array}{l}\text { Hematopoietic stem and progenitor cells } \\
\text { transplanted to a patient with HIV and acute } \\
\text { lymphoblastic leukemia }\end{array}$ & CRISPR/Cas9 & $\begin{array}{l}\text { Result in indels in CCR5 that lead to CCR5 } \\
\text { ablation }\end{array}$ & Xu et al., 2019 \\
\hline & Antiretroviral therapy in non-human primates & CRISPR/Cas9 & Eliminate proviral SIV DNA & $\begin{array}{l}\text { Mancuso et al., } \\
2020\end{array}$ \\
\hline & SupT1 cells & CRISPR/Cas12a & $\begin{array}{l}\text { Target relatively conserved HIV sequences } \\
\text { including LTRs }\end{array}$ & $\begin{array}{l}\text { Gao et al., } \\
2020\end{array}$ \\
\hline & $\begin{array}{l}\text { HIV-1 infected HEK293T and Jurkat cells, and } \\
\text { latently infected JLat10.6 cells }\end{array}$ & CRISPR/Cas13a & Target the conserved regions of HIV-1 & Yin et al., 2020 \\
\hline \multirow[t]{7}{*}{ HBV } & HepG2 cells & CRISPR/Cas9 & Lead to mutations and deletions in cccDNA & $\begin{array}{l}\text { Seeger and } \\
\text { Sohn, } 2014\end{array}$ \\
\hline & Huh7 cells, HBV persistent mouse model & CRISPR/Cas9 & $\begin{array}{l}\text { Reduce the production of HBV core and } \\
\text { surface proteins }\end{array}$ & Lin et al., 2014 \\
\hline & HepG2 and HeoG2.2.15 cells & CRISPR/Cas9 & Target the core, polymerase, and $X$ ORFs & $\begin{array}{l}\text { Ramanan et al., } \\
2015\end{array}$ \\
\hline & $\begin{array}{l}\text { Huh7 cells, HeoG2.2.15 cells, mouse model } \\
\text { carrying HBV cccDNA }\end{array}$ & CRISPR/Cas9 & Target the conserved regions of HBV & $\begin{array}{l}\text { Dong et al., } \\
2015\end{array}$ \\
\hline & HepG2 and HeoG2.2.15 cells, HBV-Tg mice & CRISPR/Cas9 & $\begin{array}{l}\text { Target the surface antigen (HBsAg)-encoding } \\
\text { region of HBV }\end{array}$ & $\begin{array}{l}\text { Zhen et al., } \\
2015\end{array}$ \\
\hline & Stable HBV cell line & CRISPR/Cas9 & Cut a 3,175-bp HBV DNA fragment & Li et al., 2017 \\
\hline & Infected hNTCP-HepG2 cells & CRISPR/Cas9 & Target the $S$ open reading frame of HBV & $\begin{array}{l}\text { Scott et al., } \\
2017\end{array}$ \\
\hline \multirow[t]{4}{*}{ HPV } & HPV-transformed cervical carcinoma cells & CRISPR/Cas9 & Target and inactivate the E6 and E7 oncogenes & $\begin{array}{l}\text { Kennedy et al., } \\
2014\end{array}$ \\
\hline & HPV-transformed cervical carcinoma cells & CRISPR/Cas9 & Disrupt the HPV16 E7 gene & $\begin{array}{l}\text { Hu Z. et al., } \\
2014\end{array}$ \\
\hline & HPV-transformed cervical carcinoma cells/mice & CRISPR/Cas9 & $\begin{array}{l}\text { Targeting promoter of HPV16 and targeting the } \\
E 6 \text { and } E 7 \text { transcripts }\end{array}$ & $\begin{array}{l}\text { Zhen et al., } \\
2014\end{array}$ \\
\hline & HPV-transformed cervical carcinoma cells & CRISPR/Cas9 & Disrupt the HPV16 E6 gene & Yu et al., 2015 \\
\hline \multirow[t]{2}{*}{ HSV-1 } & Vero cells & CRISPR/Cas9 & $\begin{array}{l}\text { Target } 12 \text { essential genes and } 2 \text { non-essential } \\
\text { genes }\end{array}$ & $\begin{array}{l}\text { van Diemen } \\
\text { et al., } 2016\end{array}$ \\
\hline & Human oligodendroglioma cells & CRISPR/Cas9 & $\begin{array}{l}\text { Indel mutations in exon } 2 \text { of the } I C P O \text { gene in } \\
\text { the HSV-1 genome }\end{array}$ & $\begin{array}{l}\text { Roehm et al., } \\
2016\end{array}$ \\
\hline \multirow[t]{2}{*}{ SARS-CoV-2 } & Synthesized fragments of SARS-CoV-2 & CRISPR/Cas13d & $\begin{array}{l}\text { Design and screen crRNAs targeting conserved } \\
\text { viral regions. Identify } 40 \text { functional crRNAs } \\
\text { targeting SARS-CoV-2 }\end{array}$ & $\begin{array}{l}\text { Abbott et al., } \\
2020\end{array}$ \\
\hline & $\begin{array}{l}\text { SARS-CoV-2 RNA genome data from } 19 \text { patients in } \\
\text { China, United States, and Australia }\end{array}$ & CRISPR/Cas13d & $\begin{array}{l}\text { In silico } 10,333 \text { guide RNAs to specifically } \\
\text { target } 10 \text { peptide-coding regions of the } \\
\text { ORF1ab and } S \text { genes }\end{array}$ & $\begin{array}{l}\text { Nguyen et al., } \\
2020\end{array}$ \\
\hline
\end{tabular}

DNA (Tebas et al., 2014). In 2019, Xu et al. (2019) used the CRISPR genome editing system to ablate the CCR5 gene ex vivo in $\mathrm{CD}^{+}{ }^{+}$hematopoietic stem and progenitor cells and successfully transplanted them back to a patient with HIV and acute lymphoblastic leukemia. The patient experienced a remission of acute lymphoblastic leukemia and showed a persistence of CCR5-ablated donor cells for no less than 19 months (Xu et al., 2019). The overall genome editing efficiency presented in their work was modest (during the 19-month engraftment period, the frequency of CCR 5 disruption in the genome of bone marrow cells ranged between 5.20\% and 8.28\%). Nonetheless, genome editing endowed the edited cells with the ability to resist HIV infection, but it could not completely eliminate the virus. Nevertheless, this study is a critical step in the translation of new technologies into clinical applications, showing the feasibility and safety of genome editing therapy for HIV in clinical practice.

Another genome editing-based strategy for preventing HIV infection is to directly destroy the integrated HIV genome in latently infected cells, and to provide long-term resistance to new viral infection, expression, and replication (Table 1; 
Liao et al., 2015b). HIV long terminal repeats (LTRs) and the env and gag genes have been targeted to reduce and eliminate HIV in different experimental settings (Wang et al., 2018). In 2014, by bioinformatic screening and off-target prediction, $\mathrm{Hu} \mathrm{Z}$. et al. (2014) identified four CRISPR/Cas9 gRNA targets in the HIV1 LTR promoter U3 region. By co-expressing the Cas9 protein and the four gRNAs, Hu Z. et al. (2014) efficiently inactivated HIV-1 gene expression in infected microglial and macrophage cells, the two particular cell types in the brain that harbor HIV1. Hu Z. et al. (2014) demonstrated that their proof-of-concept work can be applicable to T cells. Liao et al. (2015a) demonstrated that engineered human induced pluripotent stem cells that stably expressed HIV-targeted CRISPR/Cas9 could differentiate into HIV reservoir cell types and maintain the resistance to HIV1. By targeting the HIV-1 LTR promoter region, gag, and pol using sgRNA and saCas9 delivered by AAV vectors, Yin C. et al. (2017) eliminated HIV proviral DNA in various organs in three different animal models, including a humanized mouse model of chronic HIV infection. Bella et al. (2018) used a lentiviral vector to deliver a CRISPR/Cas system targeting HIV-1 LTR to infected human peripheral blood mononuclear cells to eliminate the HIV proviral DNA in humanized mouse models. By using eight male Chinese rhesus macaques with intravenous inoculation of SIV infection, a well-accepted non-human primate model of HIV infection, Mancuso et al. (2020) showed that AAV9CRISPR/Cas9 treatment targeting the $5^{\prime}$ LTR-gag and gag-3' LTR regions of the SIV genome resulted in a reduction of proviral DNA in infected blood cells and tissues. These proof-of-concept observations offer a promising step toward the elimination of HIV reservoirs in the clinic.

Besides CRISPR/Cas9, other smaller CRISPR/Cas nucleases are emerging as robust alternatives (Zetsche et al., 2015, 2017; Abudayyeh et al., 2016; Zhong et al., 2017; Paul and Montoya, 2020). Gao et al. (2020) transduced a $\mathrm{T}$ cell line with the CRISPR/Cas12a system using lentiviral vectors and observed a complete HIV sterilization using a single crRNA. Yin et al. (2020) utilized CRISPR/Cas13a to target the LTR, gag, tat, and rev regions of HIV-1 and demonstrated strong destruction of HIV1 RNA either in invading viral capsids or expressed by latent proviruses in HEK293T, Jurkat, and JLat10.6 HIV cells. However, Cas13a recognizes and cleaves RNA, and thus cannot in theory eliminate HIV DNA and achieve a complete cure.

Due to safety concerns of genome editing, most somatic genome editing experiments were done in cells and animal models. More clinical studies of somatic genome editing are still needed.

\section{Development of Genome Editing Therapy for HBV, HPV, and HSV}

More than 250 million people are living with chronic hepatitis B virus (HBV) infection (Strich and Chertow, 2019). Though effective prophylactic $\mathrm{HBV}$ vaccines are available, due to the highly stable HBV covalently closed circular DNA (cccDNA) existing in the nuclei of infected cells, effective therapies to eliminate the virus remain elusive. Recent studies showed that CRISPR/Cas could be a potential effective treatment for HBV infection (Table 1). Scott et al. (2017) successfully inactivated cccDNA in HBV-infected hNTCP-HepG2 cells by using the CRISPR/Cas system to target the $S$ open reading frame (ORF) of HBV. In the same year, Li et al. (2017) utilized CRISPR/Cas9 to cut a 2,175-bp HBV DNA that integrated into the genome of infected cells. In these experiments, AAV vectors, including AAV8 and its derivatives, showed a good tropism for liver, making them a promising vector for delivering the CRISPR/Cas anti-HBV therapy.

More than 150 different HPV serotypes can infect humans. Though most of them are harmless or only induce benign warts, a small group of the serotypes are regarded as high-risk factors for cancer. Epidemiological studies show that HPV16 and HPV18 account for about $75 \%$ of cervical cancer, while HPV31 and HPV 45 account for a further 10\%. Besides, HPVs are significantly associated with oropharyngeal and anal cancers. When HPVs overexpress the E6 and E7 proteins that inhibit cellular tumor suppressors, the risk of malignant transformation for the infected cells increases (Werness et al., 1990; Hu Z. et al., 2014; Zhen et al., 2014). It has shown that loss of E6 and E7 function induces p53 and Rb expression, respectively, and thus, induces cell cycle arrest. Inspired by this, several groups explored the possibility of utilizing CRISPR/Cas-mediated cleavage to disturb HPV E6 or E7 genes (Table 1). Kennedy et al. (2014) used CRISPR/Cas9based strategy to target the amino-terminal regions of HPV18 $E 6$ and E7 ORFs and successfully inactivated the E6 and E7 genes in cervical carcinoma cells transformed by HPV18. Hu Z. et al. (2014) demonstrated that targeting the E7 gene of HPV16 with CRISPR/Cas could destroy E7 DNA and induce apoptosis and growth inhibition of HPV-positive SiHa and Caski cells while sparing HPV-negative C33A and HEK293 cells. Besides, the disruption of E7 DNA lead not only to down-regulated E7 protein but also to up-regulated $\mathrm{pRb}$, a tumor suppressor protein ( $\mathrm{Hu}$ Z. et al., 2014). Zhen et al. (2014) transduced the CRISPR/Cas9 system targeting the promoter and transcripts of HPV16 E6/E7 into HPV16-positive SiHa cells and observed increased levels of p53 and p21 proteins. These efforts showed genome editing as an effective path to eliminate HPV DNA, down-regulate the expression of the two oncogenes E6 and E7, and up-regulate tumor suppressors $p 53$ and $p 21$, suggesting the promise of CRISPR/Cas for treating HPV infectious diseases (Hu Z. et al., 2014; Kennedy et al., 2014; Zhen et al., 2014; Yu et al., 2015).

HSV is another virus that spreads around the world. van Diemen et al. (2016) reduced HSV-1 replication in Vero cells by targeting 14 different genes using the CRISPR/Cas9 system. Targeting multiple genes using genome editing shows a greater efficiency of HSV-1 elimination than targeting single genes (van Diemen et al., 2016). Roehm et al. (2016) suppressed HSV1 replication in human oligodendroglioma cells by inducing mutations into the ICP0, ICP4, and ICP27 genes that are important for viral replication.

In addition to the above-mentioned cases, proof-of-concept laboratory studies have also shown that genome editing technologies can target and destroy other viruses including lymphocytic choriomeningitis virus, influenza A virus, and vesicular stomatitis virus (Freije et al., 2019). Such versatility of genome editing-based antiviral treatment is perhaps best 
exemplified by the rapid research progress toward the prevention of SARS-CoV-2 infection using CRISPR, which deserves a separate discussion below.

\section{Development of Genome Editing Therapy for SARS-CoV-2}

SARS-CoV-2 has been rapidly spreading around the world and has caused more than 2.5 million deaths at the time of writing. While several vaccines have shown good efficacies in preventing COVID-19, vaccine supply shortages, emerging mutant variants around the world, inadequate immune response in immunocompromised individuals, and rare side effects still leave hundreds of millions of people exposed to the risk of contracting SARS-CoV-2. Available small molecule antiviral drugs have limited effects on SARS-CoV-2. Although monoclonal antibodies are cleared by the US FDA for emergency use, they are quite expensive and are only authorized for mild to moderate COVID-19 cases and may be associated with worse outcomes when administered to severe cases that require hospitalization. Thus, new anti-viral treatments for SARS-CoV-2 are urgently needed.

Recently, the CRISPR/Cas13 system has been under the spotlight since it targets RNA and shows a great potential in treating RNA viral infectious diseases, including COVID19. Researchers have used CRISPR/Cas13-based technology to develop novel antiviral drugs to combat SARS-CoV-2 (Table 1; Abbott et al., 2020; Nguyen et al., 2020). By targeting highly conserved regions of the SARS-CoV-2 genome using a gRNA, Cas 13 can cut and clear the viral RNA genome. The action of the CRISPR system can be organ specific by leveraging AAV serotypes that are specific to the lung. Besides, researchers can apply multiple gRNAs to ensure successful targeting of the virus even if part of it mutates. To help develop a CRISPR/Cas13d system that specifically eliminates SARS-CoV-2 genome, Nguyen et al. (2020) designed 10,333 gRNAs to target ten coding regions of the ORF1ab and $S$ genes in the SARS-CoV-2 genome and proposed AAV to be the vector to simultaneously deliver up to three gRNAs targeting different coding regions of SARSCoV-2. The authors claimed that the gRNAs did not affect the human transcriptome, though experimental data should be added to support the efficacy and safety of their system (Nguyen et al., 2020). Abbott et al. (2020) established a prophylactic antiviral CRISPR in human cells (PAC-MAN) strategy that uses CRISPR/Cas13d to degrade viral sequences. The PAC-MAN strategy proved to be capable of inhibiting SARS-CoV-2 fragment expression as well as influenza A virus infection in human lung epithelial cells (Abbott et al., 2020). Additionally, they utilized bioinformatics analysis to predict groups of CRISPRassociated RNAs to target all sequenced coronaviruses, though the effectiveness and in vivo safety of this strategy need to be examined before putting it into clinical use (Abbott et al., 2020). To expand their work, Lin et al. (2021) applied the PAC-MAN strategy to a broad spectrum of human- or livestock-tropic RNA viruses. The in silico analysis showed that a minimal set of 14 crRNAs can target $>90 \%$ of human-tropic viral genomes. Furthermore, by co-transfecting plasmids containing one of five predicted crRNAs and SARS-CoV-2 reporters into A549 lung epithelial cells, Lin et al. (2021) validated the PAC-MAN strategy. Specifically, they observed that the decrease in the percentage of SARS-Cov-2 reporter-positive cells correlated significantly with the predicted targeting efficiency score of crRNAs and with the viral RNA abundance. These results validated their prediction algorithm for the targeting efficiency of crRNAs for the viral genome (Lin et al., 2021).

While individual crRNA showed an ability to target almost $99 \%$ of SARS-CoV-2 isolates, the pooling of the five experimentally validated crRNAs could target all of the SARSCoV-2 variants as identified in GISAID on February 3, 2021 (Lin et al., 2021). Moreover, Lin et al. (2021) have rolled out an online resource ${ }^{1}$ for the use of CRISPR/Cas13 to target RNA viruses, which when combined with rapid field-deployable virus sequencing (Bi et al., 2021) could greatly speed up the design of genome editing tools for combating emerging viruses. These proof-of-principle studies highlight the versatility and flexibility of CRISPR as an intracellular defense against many types of RNA viruses, including but not limited to SARS-CoV-2.

\section{CONCLUSION AND PERSPECTIVES}

The flexible characteristic of genome editing technologies, especially the CRISPR/Cas system, gives it an inherent advantage when dealing with the fast evolution of mutations in viral pathogens. Though clinically approved genome editing therapies are not yet available, early clinical trials are ongoing. Safety problems, including immunogenicity and mutagenesis, still need investigation. Recent reports showed that DNA repair following the double strand breaks induced by the CRISPR/Cas system can cause unintended mutations including large structural variations (Kosicki et al., 2018; Bi et al., 2020). Furthermore, Haapaniemi et al. (2018) showed that CRISPR/Cas9 can induce p53-mediated DNA damage response and leads to a selection against p53 proficient cells. Since p53-mediated mechanisms are key to maintaining genome stability, potential tumorigenesis originated from genome-edited cells remains a concern and needs to be further examined.

Efficient and safe delivery of genome editing technologies remains a challenge, especially for in vivo applications. To this end, Yin H. et al. (2017) developed an optimal set of chemical modifications in sgRNAs to maintain or enhance genomeediting efficiency for in vivo applications. A single intravenous injection of the enhanced sgRNAs with CRISPR/Cas9-based lipid nanoparticle formulations into mice induced $>80 \%$ disruption of Pcsk9 in the liver and achieved undetectable levels of Pcsk9 in the serum (Yin H. et al., 2017). Alsaiari et al. (2018) developed nanoscale zeolitic imidazole frameworks (ZIFs) to deliver CRISPR/Cas9 to mammalian cells as nanoparticles and showed CRISPR/Cas-ZIFs are biocompatible and offer efficient co-delivery of Cas9 protein and sgRNA. Mangeot et al. (2019) developed a vehicle called nanoblades by loading Cas9-sgRNA ribonucleoproteins onto engineered murine leukemia virus-like particles. Nanoblades targeting $\mathrm{Hpd}$ achieved between $7 \%$ and

\footnotetext{
${ }^{1}$ crispr-pacman.stanford.edu
} 
$13 \%$ targeting efficiency in mouse liver 2 weeks after retroorbital injection and showed no signs of morbidity (Mangeot et al., 2019). The choice of vector is still under discussion. As mentioned before, AAV2 showed insertional mutagenesis in human hepatocellular carcinomas (Nault et al., 2015). Besides, the current evidence clearly demonstrates that AAV vectors can trigger innate and adaptive immune responses, leading to transgene loss (Mingozzi and High, 2013; Ronzitti et al., 2020; Samelson-Jones et al., 2020). Vector design and the total dose of AAVs need to be optimized to decrease the immune-mediated toxicity before clinical use (Ling Li et al., 2015; Ronzitti et al., 2020).

Ethical concern of human genome editing is a hotly debated issue (Isasi et al., 2016). Genome editing therapies for viral pathogens carry the risk of altering the human genome. The current consensus recommendation is that genome editing in somatic cells is acceptable if the goal is to treat severe diseases, while germline editing is strongly discouraged (National Academies of Sciences, 2017; National Academy of Sciences, 2020). In this sense, genome editing therapies for viral infectious diseases are likely to encounter less ethical issues as they only target somatic cells.

In sum, exciting advances have been made in the development of genome editing as treatment for viral infectious diseases, but significant challenges lie before clinical translation of the research. Some of these challenges, such as safety and efficiency of CRISPR, are common to genome editing technologies and are being addressed by the genome editing field, while

\section{REFERENCES}

Abbott, T. R., Dhamdhere, G., Liu, Y., Lin, X., Goudy, L., Zeng, L., et al. (2020). Development of CRISPR as an Antiviral Strategy to Combat SARS-CoV-2 and Influenza. Cell 181, 865.e-876.e.

Abudayyeh, O. O., Gootenberg, J. S., Essletzbichler, P., Han, S., Joung, J., Belanto, J. J., et al. (2017). RNA targeting with CRISPR-Cas13. Nature 550, 280-284.

Abudayyeh, O. O., Gootenberg, J. S., Konermann, S., Joung, J., Slaymaker, I. M., Cox, D. B., et al. (2016). C2c2 is a single-component programmable RNAguided RNA-targeting CRISPR effector. Science 353:aaf5573. doi: 10.1126/ science.aaf5573

Alsaiari, S. K., Patil, S., Alyami, M., Alamoudi, K. O., Aleisa, F. A., Merzaban, J. S., et al. (2018). Endosomal Escape and Delivery of CRISPR/Cas9 Genome Editing Machinery Enabled by Nanoscale Zeolitic Imidazolate Framework. J. Am. Chem. Soc. 140, 143-146. doi: 10.1021/jacs.7b11754

Alwin, S., Gere, M. B., Guhl, E., Effertz, K., Barbas, C. F., Segal, D. J., et al. (2005). Custom Zinc-Finger Nucleases for Use in Human Cells. Mole. Ther. 12, 610-617.

Anzalone, A. V., Koblan, L. W., and Liu, D. R. (2020). Genome editing with CRISPR-Cas nucleases, base editors, transposases and prime editors. Nat. Biotechnol. 38, 824-844. doi: 10.1038/s41587-020-0561-9

Athanasopoulos, T., Munye, M. M., and Yáñez-Muñoz, R. J. Y. (2017). Nonintegrating Gene Therapy Vectors. Hematol. Oncol. Clin. North Am. 31, 753-770. doi: 10.1016/j.hoc.2017.06.007

Bella, R., Kaminski, R., Mancuso, P., Young, W.-B., Chen, C., Sariyer, R., et al. (2018). Removal of HIV DNA by CRISPR from Patient Blood Engrafts in Humanized Mice. Mole. Ther. 12, 275-282. doi: 10.1016/j.omtn.2018.05.021

Bi, C., Ramos-Mandujano, G., Tian, Y., Hala, S., Xu, J., Mfarrej, S., et al. (2021). Simultaneous Detection and Mutation Surveillance of SARS-CoV-2 and coinfections of multiple respiratory viruses by Rapid field-deployable sequencing. medRxiv doi: 10.1101/2020.06.12.20129247 others require a commitment to progress from proof-ofconcept studies to preclinical animal models and ultimately to clinical trials. With the COVID-19 pandemic raging through the world and causing human tragedies and economic devastations globally, now may just be the right time to commit to make genome editing therapy for infectious disease a clinical reality.

\section{AUTHOR CONTRIBUTIONS}

YZ reviewed the literature and drafted the manuscript. ML revised the manuscript and supervised the study. YZ and ML conceived the study. Both authors contributed to the article and approved the submitted version.

\section{FUNDING}

This work was supported by grants awarded to ML under award numbers BAS/1/1080-01 and REI/1/4742-01 by KAUST Office of Sponsored Research (OSR).

\section{ACKNOWLEDGMENTS}

We thank Baolei Yuan and Xuan Zhou for helpful discussions and Deng Luo for proofreading.

Bi, C., Wang, L., Yuan, B., Zhou, X., Li, Y., Wang, S., et al. (2020). Long-read individual-molecule sequencing reveals CRISPR-induced genetic heterogeneity in human ESCs. Genome Biol. 21:213.

Bitinaite, J., Wah, D. A., Aggarwal, A. K., and Schildkraut, I. (1998). FokI dimerization is required for DNA cleavage. Proc. Natl. Acad. Sci. 95:10570. doi: 10.1073/pnas.95.18.10570

Blankson, J. N., Siliciano, J. D., and Siliciano, R. F. (2014). Finding a cure for human immunodeficiency virus-1 infection. Infect. Dis. Clin. North Am. 28, 633-650. doi: 10.1016/j.idc.2014.08.007

Boch, J., Scholze, H., Schornack, S., Landgraf, A., Hahn, S., Kay, S., et al. (2009). Breaking the code of DNA binding specificity of TAL-type III effectors. Science 326, 1509-1512. doi: 10.1126/science.1178811

Deltcheva, E., Chylinski, K., Sharma, C. M., Gonzales, K., Chao, Y., Pirzada, Z. A., et al. (2011). CRISPR RNA maturation by trans-encoded small RNA and host factor RNase III. Nature 471, 602-607. doi: 10.1038/nature09886

Dong, C., Qu, L., Wang, H., Wei, L., Dong, Y., and Xiong, S. (2015). Targeting hepatitis B virus cccDNA by CRISPR/Cas9 nuclease efficiently inhibits viral replication. Antiviral. Res. 118, 110-117. doi: 10.1016/j.antiviral.2015.03.015

Freije, C. A., Myhrvold, C., Boehm, C. K., Lin, A. E., Welch, N. L., Carter, A., et al. (2019). Programmable inhibition and detection of RNA viruses using Cas13. Mol. Cell 76, 826-837.e11.

Gao, Z., Fan, M., Das, A. T., Herrera-Carrillo, E., and Berkhout, B. (2020). Extinction of all infectious HIV in cell culture by the CRISPR-Cas12a system with only a single crRNA. Nucleic Acids Res. 48, 5527-5539. doi: 10.1093/nar/ gkaa226

Garneau, J. E., Dupuis, M., Villion, M., Romero, D. A., Barrangou, R., Boyaval, P., et al. (2010). The CRISPR/Cas bacterial immune system cleaves bacteriophage and plasmid DNA. Nature 468, 67-71. doi: 10.1038/nature09523

Gupta, R. K., Abdul-Jawad, S., McCoy, L. E., Mok, H. P., Peppa, D., Salgado, M., et al. (2019). Olavarria, HIV-1 remission following CCR5 $\Delta 32 / \Delta 32$ haematopoietic stem-cell transplantation. Nature 568, 244-248. 
Haapaniemi, E., Botla, S., Persson, J., Schmierer, B., and Taipale, J. (2018). CRISPRCas 9 genome editing induces a p53-mediated DNA damage response. Nat. Med. 24, 927-930. doi: 10.1038/s41591-018-0049-z

Hanlon, K. S., Kleinstiver, B. P., Garcia, S. P., Zaborowski, M. P., Volak, A., Spirig, S. E., et al. (2019). High levels of AAV vector integration into CRISPR-induced DNA breaks. Nat. Comm. 10:4439.

Hu, W., Kaminski, R., Yang, F., Zhang, Y., Cosentino, L., Li, F., et al. (2014). RNA-directed gene editing specifically eradicates latent and prevents new HIV-1 infection. Proc. Natl. Acad. Sci. 111, 11461-11466. doi: 10.1073/pnas. 1405186111

Hu, Z., Yu, L., Zhu, D., Ding, W., Wang, X., Zhang, C., et al. (2014). Disruption of HPV16-E7 by CRISPR/Cas System Induces Apoptosis and Growth Inhibition in HPV16 Positive Human Cervical Cancer Cells. BioMed. Res. Internat. 2014:612823.

Isasi, R., Kleiderman, E., and Knoppers, B. M. (2016). Editing policy to fit the genome? Science 351:337. doi: 10.1126/science.aad6778

Ishino, Y., Shinagawa, H., Makino, K., Amemura, M., and Nakata, A. (1987). Nucleotide sequence of the iap gene, responsible for alkaline phosphatase isozyme conversion in Escherichia coli, and identification of the gene product. J. Bacteriol. 169, 5429-5433. doi: 10.1128/jb.169.12.5429-5433.1987

Jiang, F., and Doudna, J. A. (2017). CRISPR-Cas9 Structures and Mechanisms. Ann. Rev. Biophys. 46, 505-529. doi: 10.1146/annurev-biophys-062215-010822

Kennedy, E. M., Kornepati, A. V. R., Goldstein, M., Bogerd, H. P., Poling, B. C., Whisnant, A. W., et al. (2014). Inactivation of the Human Papillomavirus E6 or E7 Gene in Cervical Carcinoma Cells by Using a Bacterial CRISPR/Cas RNA-Guided Endonuclease. J. Virol. 88, 11965-11972. doi: 10.1128/jvi.018 79-14

Kim, Y. G., Cha, J., and Chandrasegaran, S. (1996). Hybrid restriction enzymes: zinc finger fusions to Fok I cleavage domain. Proc. Natl. Acad. Sci. U S A 93, 1156-1160. doi: 10.1073/pnas.93.3.1156

King, D. A., Peckham, C., Waage, J. K., Brownlie, J., and Woolhouse, M. E. J. (2006). Infectious Diseases: Preparing for the Future. Science 313:1392.

Kosicki, M., Tomberg, K., and Bradley, A. (2018). Repair of double-strand breaks induced by CRISPR-Cas9 leads to large deletions and complex rearrangements. Nat. Biotechnol. 36, 765-771. doi: 10.1038/nbt.4192

Kulkarni, T. A., Bade, A. N., Sillman, B., Shetty, B. L. D., Wojtkiewicz, M. S., Gautam, N., et al. (2020). A year-long extended release nanoformulated cabotegravir prodrug. Nat. Material. 19, 910-920. doi: 10.1038/s41563-0200674-z

Lander, E. S. (2016). The Heroes of CRISPR. Cell 164, 18-28. doi: 10.1016/j.cell. 2015.12.041

Li, H., Sheng, C., Wang, S., Yang, L., Liang, Y., Huang, Y., et al. (2017). Removal of Integrated Hepatitis B Virus DNA Using CRISPR-Cas9. Front. Cell. Infect. Microbiol. 2017:7.

Li, M., Suzuki, K., Kim, N. Y., Liu, G. H., and Izpisua Belmonte, J. C. (2014). A cut above the rest: targeted genome editing technologies in human pluripotent stem cells. J. Biol. Chem. 289, 4594-4599. doi: 10.1074/jbc.r113.488247

Liao, H. K., Gu, Y., Diaz, A., Marlett, J., Takahashi, Y., Li, M., et al. (2015a). Use of the CRISPR/Cas9 system as an intracellular defense against HIV-1 infection in human cells. Nat. Comm. 6:6413.

Liao, H. K., Li, M., Martinez Martinez, L., and Izpisua Belmonte, J. C. (2015b). Stem cell, CRISPR and HIV. Cell Cycle 14, 1991-1992. doi: 10.1080/15384101.2015. 1046791

Lin, S.-R., Yang, H.-C., Kuo, Y.-T., Liu, C.-J., Yang, T.-Y., Sung, K.-C., et al. (2014). The CRISPR/Cas9 System Facilitates Clearance of the Intrahepatic HBV Templates In Vivo. Mole. Ther. Nucleic Acids 3:e186. doi: 10.1038/mtna. 2014.38

Lin, X., Liu, Y., Chemparathy, A., Pande, T., La Russa, M., and Qi, L. S. (2021). A comprehensive analysis and resource to use CRISPR-Cas13 for broad-spectrum targeting of RNA viruses. Cell Reports Medicine 2021:100245. doi: 10.1016/j. Xcrm.2021.100245

Ling Li, Z.-Y. H., Xia-Wei, W., Guang-Ping, G., and Yu-Quan, W. (2015). Challenges in CRISPR/CAS9 Delivery: Potential Roles of Nonviral Vectors. Hum. Gene Ther 26, 452-462. doi: 10.1089/hum.2015.069

Mancuso, P., Chen, C., Kaminski, R., Gordon, J., Liao, S., Robinson, J. A., et al. (2020). CRISPR based editing of SIV proviral DNA in ART treated non-human primates. Nat. Comm. 11:6065.
Mangeot, P. E., Risson, V., Fusil, F., Marnef, A., Laurent, E., Blin, J., et al. (2019). Genome editing in primary cells and in vivo using viral-derived Nanoblades loaded with Cas9-sgRNA ribonucleoproteins. Nat. Comm. 10:45.

Mingozzi, F., and High, K. A. (2013). Immune responses to AAV vectors: overcoming barriers to successful gene therapy. Blood 122, 23-36. doi: 10.1182/ blood-2013-01-306647

National Academies of Sciences (2017). National Academy of Sciences Engineering, and Medicine, Human genome editing: science, ethics, and governance. Washington, DC: The National Academies Press.

National Academy of Sciences (2020). The Royal Society; National Academy of Sciences; National Academy of Medicine; International Commission on the Clinical Use of Human Germline Genome Editing, Heritable Human Genome Editing. Washington, DC: National Academies Press.

Nault, J.-C., Datta, S., Imbeaud, S., Franconi, A., Mallet, M., Couchy, G., et al. (2015). Recurrent AAV2-related insertional mutagenesis in human hepatocellular carcinomas. Nat. Genet. 47, 1187-1193. doi: 10.1038/ng.3389

Nguyen, T. M., Zhang, Y., and Pandolfi, P. P. (2020). Virus against virus: a potential treatment for 2019-nCov (SARS-CoV-2) and other RNA viruses. Cell Res. 30, 189-190. doi: 10.1038/s41422-020-0290-0

Paul, B., and Montoya, G. (2020). CRISPR-Cas12a: Functional overview and applications. Biomed. J. 43, 8-17. doi: 10.1016/j.bj.2019.10.005

Porteus, M. H., and Baltimore, D. (2003). Chimeric Nucleases Stimulate Gene Targeting in Human Cells. Science 300:763. doi: 10.1126/science.1078395

Porteus, M. H., and Carroll, D. (2005). Gene targeting using zinc finger nucleases. Nat. Biotechnol. 23, 967-973. doi: 10.1038/nbt1125

Pujanandez, L. (2020). Clearing a path for an HIV cure. Science 367:1086.

Ramanan, V., Shlomai, A., Cox, D. B. T., Schwartz, R. E., Michailidis, E., Bhatta, A., et al. (2015). CRISPR/Cas9 cleavage of viral DNA efficiently suppresses hepatitis B virus. Sci. Rep. 5:10833.

Roehm, P. C., Shekarabi, M., Wollebo, H. S., Bellizzi, A., He, L., Salkind, J., et al. (2016). Inhibition of HSV-1 Replication by Gene Editing Strategy. Sci. Rep. 6:23146.

Ronzitti, G., Gross, D.-A., and Mingozzi, F. (2020). Human Immune Responses to Adeno-Associated Virus (AAV) Vectors. Front. Immunol. 2020:11.

Samelson-Jones, B. J., Finn, J. D., Favaro, P., Wright, J. F., and Arruda, V. R. (2020). Timing of Intensive Immunosuppression Impacts Risk of Transgene Antibodies after AAV Gene Therapy in Nonhuman Primates. Mole. Ther. Methods Clin. Dev. 17, 1129-1138. doi: 10.1016/j.omtm.2020.05.001

Scott, T., Moyo, B., Nicholson, S., Maepa, M. B., Watashi, K., Ely, A., et al. (2017). ssAAVs containing cassettes encoding SaCas9 and guides targeting hepatitis B virus inactivate replication of the virus in cultured cells. Sci. Rep. $7: 7401$.

Seeger, C., and Sohn, J. A. (2014). Targeting Hepatitis B Virus With CRISPR/Cas9. Mole. Ther. Nucleic Acids 3:e216. doi: 10.1038/mtna.2014.68

Strich, J. R., and Chertow, D. S. (2019). CRISPR-Cas Biology and Its Application to Infectious Diseases. J. Clin. Microbiol. 57, e1307-e1318.

Szczepek, M., Brondani, V., Büchel, J., Serrano, L., Segal, D. J., and Cathomen, T. (2007). Structure-based redesign of the dimerization interface reduces the toxicity of zinc-finger nucleases. Nature Biotechnology 25, 786-793. doi: 10. $1038 /$ nbt1317

Tebas, P., Stein, D., Tang, W. W., Frank, I., Wang, S. Q., Lee, G., et al. (2014). Gene Editing of CCR5 in Autologous CD4 T Cells of Persons Infected with HIV. N. Engl. J. Med. 370, 901-910.

Trickey, A., May, M. T., Vehreschild, J.-J., Obel, N., Gill, M. J., Crane, H. M., et al. (2017). Survival of HIV-positive patients starting antiretroviral therapy between 1996 and 2013: a collaborative analysis of cohort studies. Lancet HIV 4, e349-e356.

Urnov, F. D., Rebar, E. J., Holmes, M. C., Zhang, H. S., and Gregory, P. D. (2010). Genome editing with engineered zinc finger nucleases. Nat. Rev. Genet. 11, 636-646. doi: 10.1038/nrg2842

van Diemen, F. R., Kruse, E. M., Hooykaas, M. J., Bruggeling, C. E., Schürch, A. C., van Ham, P. M., et al. (2016). CRISPR/Cas9-Mediated Genome Editing of Herpesviruses Limits Productive and Latent Infections. PLoS Pathog. 12:e1005701. doi: 10.1371/journal.ppat.1005701

Wang, G., Zhao, N., Berkhout, B., and Das, A. T. (2018). CRISPR-Cas based antiviral strategies against HIV-1. Virus Res. 244, 321-332. doi: 10.1016/j. virusres.2017.07.020 
Werness, B. A., Levine, A. J., and Howley, P. M. (1990). Association of human papillomavirus types 16 and 18 E6 proteins with p53. Science 248, 76-79. doi: 10.1126/science. 2157286

Xu, L., Wang, J., Liu, Y., Xie, L., Su, B., Mou, D., et al. (2019). CRISPR-Edited Stem Cells in a Patient with HIV and Acute Lymphocytic Leukemia. N. Engl. J. Med. 381, 1240-1247. doi: 10.1056/nejmoa1817426

Yin, C., Zhang, T., Qu, X., Zhang, Y., Putatunda, R., Xiao, X., et al. (2017). In Vivo Excision of HIV-1 Provirus by saCas9 and Multiplex Single-Guide RNAs in Animal Models. Mole. Ther. 25, 1168-1186. doi: 10.1016/j.ymthe.2017.03.012

Yin, H., Song, C.-Q., Suresh, S., Wu, Q., Walsh, S., Rhym, L. H., et al. (2017). Structure-guided chemical modification of guide RNA enables potent non-viral in vivo genome editing. Nat. Biotechnol. 35, 1179-1187. doi: 10.1038/nbt.4005

Yin, L., Zhao, F., Sun, H., Wang, Z., Huang, Y., Zhu, W., et al. (2020). CRISPRCas13a Inhibits HIV-1 Infection. Mole. Ther. Acids 21, 147-155. doi: 10.1016/j. omtn.2020.05.030

Yu, L., Wang, X., Zhu, D., Ding, W., Wang, L., Zhang, C., et al. (2015). Disruption of human papillomavirus 16 E6 gene by clustered regularly interspaced short palindromic repeat/Cas system in human cervical cancer cells. Onco Targets Ther. 8, 37-44. doi: 10.2147/ott.s64092

Zetsche, B., Heidenreich, M., Mohanraju, P., Fedorova, I., Kneppers, J., DeGennaro, E. M., et al. (2017). Multiplex gene editing by CRISPR-Cpf1 using a single crRNA array. Nat. Biotechnol. 35, 31-34. doi: 10.1038/nbt.3737

Zetsche, B., Jonathan, S., Gootenberg, O., Abudayyeh, O., Ian, M., Kira, S., et al. (2015). Cpf1 Is a Single RNA-Guided Endonuclease of a
Class 2 CRISPR-Cas System. Cell 163, 759-771. doi: 10.1016/j.cell.2015. 09.038

Zhen, S., Hua, L., Liu, Y. H., Gao, L. C., Fu, J., Wan, D. Y., et al. (2015). Harnessing the clustered regularly interspaced short palindromic repeat (CRISPR)/CRISPR-associated Cas9 system to disrupt the hepatitis B virus. Gene Therapy 22, 404-412. doi: 10.1038/gt.2015.2

Zhen, S., Hua, L., Takahashi, Y., Narita, S., Liu, Y.-H., and Li, Y. (2014). In vitro and in vivo growth suppression of human papillomavirus 16-positive cervical cancer cells by CRISPR/Cas9. Biochem. Biophys. Res. Commun. 450, 1422-1426. doi: 10.1016/j.bbrc.2014.07.014

Zhong, G., Wang, H., Li, Y., Tran, M. H., and Farzan, M. (2017). Cpf1 proteins excise CRISPR RNAs from mRNA transcripts in mammalian cells. Nat. Chem. Biol. 13, 839-841. doi: 10.1038/nchembio.2410

Conflict of Interest: The authors declare that the research was conducted in the absence of any commercial or financial relationships that could be construed as a potential conflict of interest.

Copyright (c) 2021 Zhang and Li. This is an open-access article distributed under the terms of the Creative Commons Attribution License (CC BY). The use, distribution or reproduction in other forums is permitted, provided the original author(s) and the copyright owner(s) are credited and that the original publication in this journal is cited, in accordance with accepted academic practice. No use, distribution or reproduction is permitted which does not comply with these terms. 